\title{
SOME FACTORS INFLUENCING THE PLATEAU- FORMATION IN CO-TREATED OR Ni-TREATED SINGLE MYELINATED NERVE FIBRES
}

\author{
Hidehiko TAKAHASHI, Sadao UsUdA \\ and Sadahiro EHARA* \\ Department of Physiology, Tokyo Medical College
}

In the previous papers, TAKAHASHI et al. ${ }^{1,2)}$ reported the effects of divalent transition metals, such as $\mathrm{Co}$ and $\mathrm{Ni}$ on the excitation mechanism of the myelinated nerve fibre. According to their observations, the main features of the effects of these metals are (a) increase in the rheobase, (b) enormous prolongation of the action current or potential forming a 'plateau' and (c) formation of a marked 'shoulder' in the action potential from which the membrane potential falls rapidly toward the resting level. The remarkable plateau-forming action of nickel ion upon the action potential of the myelinated nerve fibre was also observed by BRADY et al. ${ }^{3)}$

The main purpose of the present investigation was to examine the effects of some agents on the plateau formation of the action current in Co-treated or Ni-treated myelinated nerve fibres, in order to elucidate some characteristics of the excitation mechanism of the nodal membrane.

\section{METHODS}

Experiments were carried out exclusively on single large myelinated nerve fibres dissected from Japanese toad's sciatic nerves under a binocular microscope. Experimental procedures and electrophysiological techniques utilized in this study were previously described precisely2).

\section{RESULTS}

The effect of $\mathrm{Co}$ or $\mathrm{Ni}$ ion upon the action current and the rheobase varied considerably from preparation to preparation. However, their actions on any preparation were stable and remained very constant for a few hours. Therefore, quantitative investigations of the effects of some agents on the plateau of the action current produced by them were possible.

Received for publication May 11, 1962.

* 高橋日出彦, 桼田貞夫, 江原貞博 
After their actions had fully developed, various ionic components of surrounding media were changed in their concentrations or some chemical agents were added to the media and their effects on the plateau were observed. The essential features of their effects were the same in Ni-treated nerve fibre as in Co-treated ones. Consequently, the description in the text was mainly limited to the case of Co-treated nerve fibres.

$\mathrm{Na}$ ion and plateau. In Co-treated nerve fibres immersed in normal Ringer, complete replacement of sodium with lithium induced no change in the spike height of the action current, but it produced some increase in the rheobase and a slight curtailment of the plateau. The curtailment was accompanied by

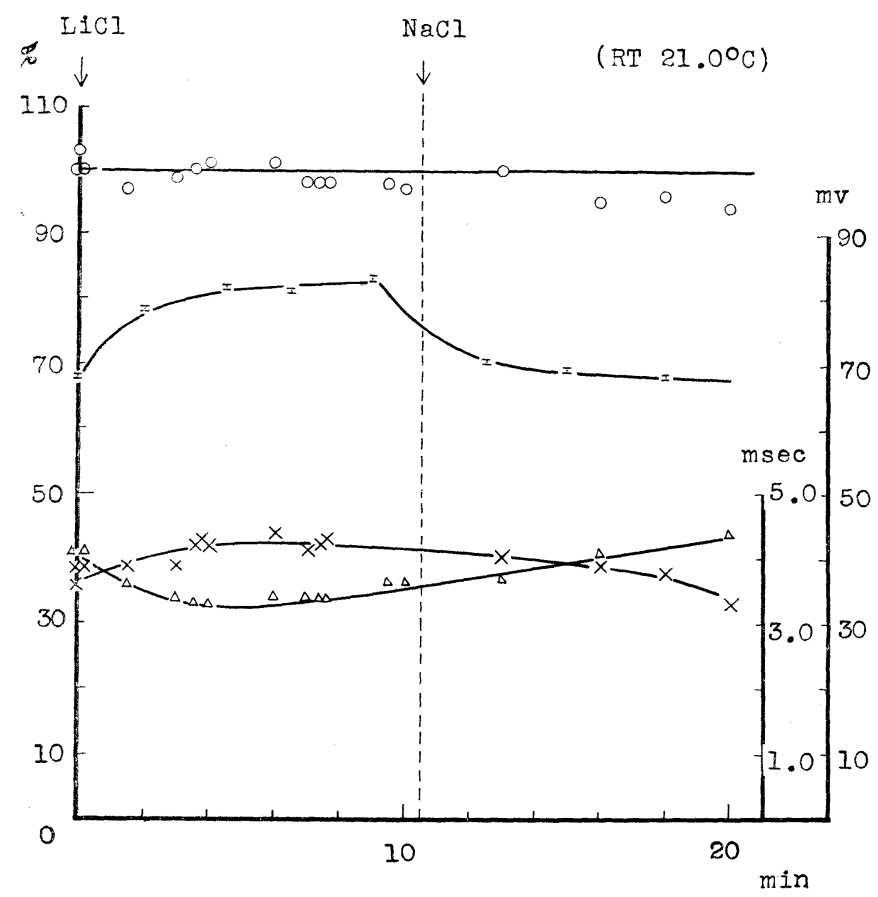

FIG. 1. Effects of the complete replacement of $\mathrm{NaCl}$ with $\mathrm{LiCl}$ in a Co-treated nerve fibre. At first $\mathrm{CoCl}_{2}\left(10^{-3} \mathrm{~mole} / 1\right)$ was added to the medium and then $\mathrm{NaCl}$ of the medium was completely replaced with LiCl. At last, normal Ringer containing $\mathrm{CoCl}_{2}$ was again applied. In this and all the following figures, the same symbols for the rheobase (I), the spike heigh $(\bigcirc)$, the duration of the action current $(\Delta)$ and the shoulder height $(x)$ were used.

a slight increase in the shoulder height. These changes produced by the replacement were completely reversible and disappeared when the fibres were again immersed in normal Ringer (FIG. 1).

When $\mathrm{NaCl}$ in the media was replaced by sucrose maintaining the osmolarity 
constant, the plateau of the action current was curtailed. The duration of the plateau or of the whole action current was decreased linearly with proportion to the logarithm of sodium chloride concentration in the external media, even though the spike height was not necessarily decreased linearly.

On the other hand, the curtailment of the plateau duration produced by the replacement of $\mathrm{NaCl}$ with Choline Chloride deviated widely from the linear relationship at low sodium concentrations. This deviation was probably due to a favourable effect on the plateau formation of high concentrated choline chloride (FIG. 2). It is also seen in FIG. 2 that increase in the external sodium concentration prolongs the plateau duration further.

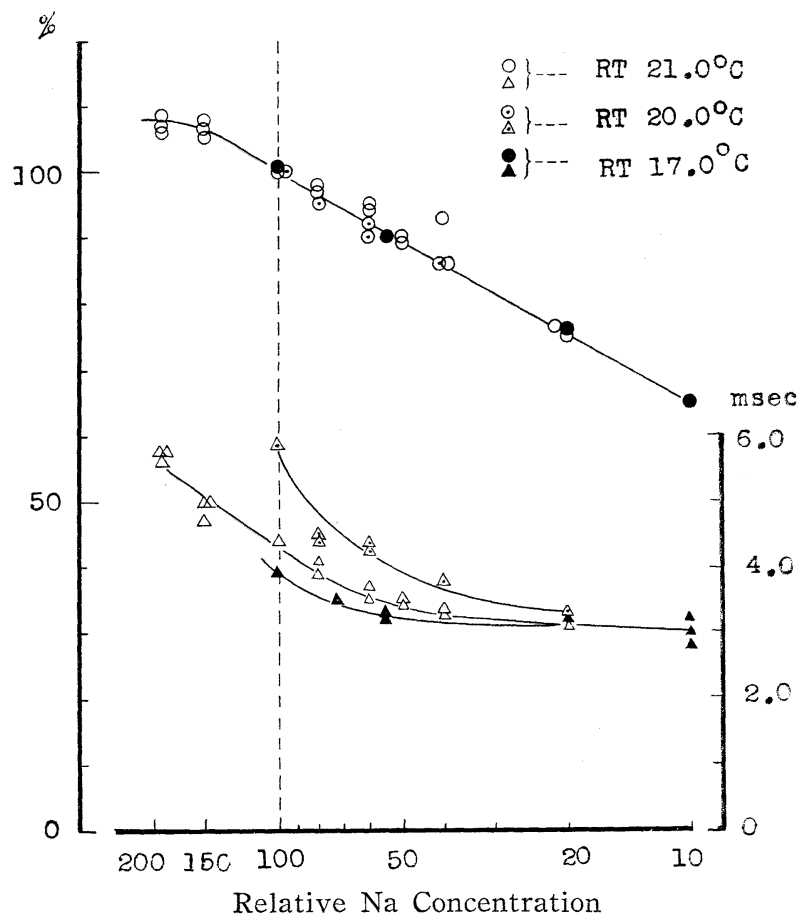

FIG. 2. Relationship between the spike height, the duration of the action current and the external sodium concentration. In this figure, changes in the spike height and the duration of the action current of three Co-treated fibres were plotted against the logarithm of the external sodium concentration. $\mathrm{NaCl}$ of the media was replaced with choline chloride. Na concentration is expressed as multiple of concentration of standard solution (arbitrarily as 100).

Subsequently, the relationship between the peak value, the plateau duration of the action potential and sodium concentration in Co-treated fibres was investigated. In such investigations, the action potentials of nerve fibres were led by TASAKI and FRANK's method ${ }^{4}$ and $\mathrm{NaCl}$ of the media was replaced with 
sucrose. In FIG. 3 a typical example of such experiments is illustrated. In this experiment, the maximum value of the action potential was $119 \mathrm{mv}$ in normal Ringer. The amplitude of action potential was increased to $132 \mathrm{mv}$. and remarkable plateau was formed in the action potential after application of $10^{-3}$ mole $/ 1 \mathrm{CoCl}_{2}$. Both the maximum voltage of action potentials and the plateau

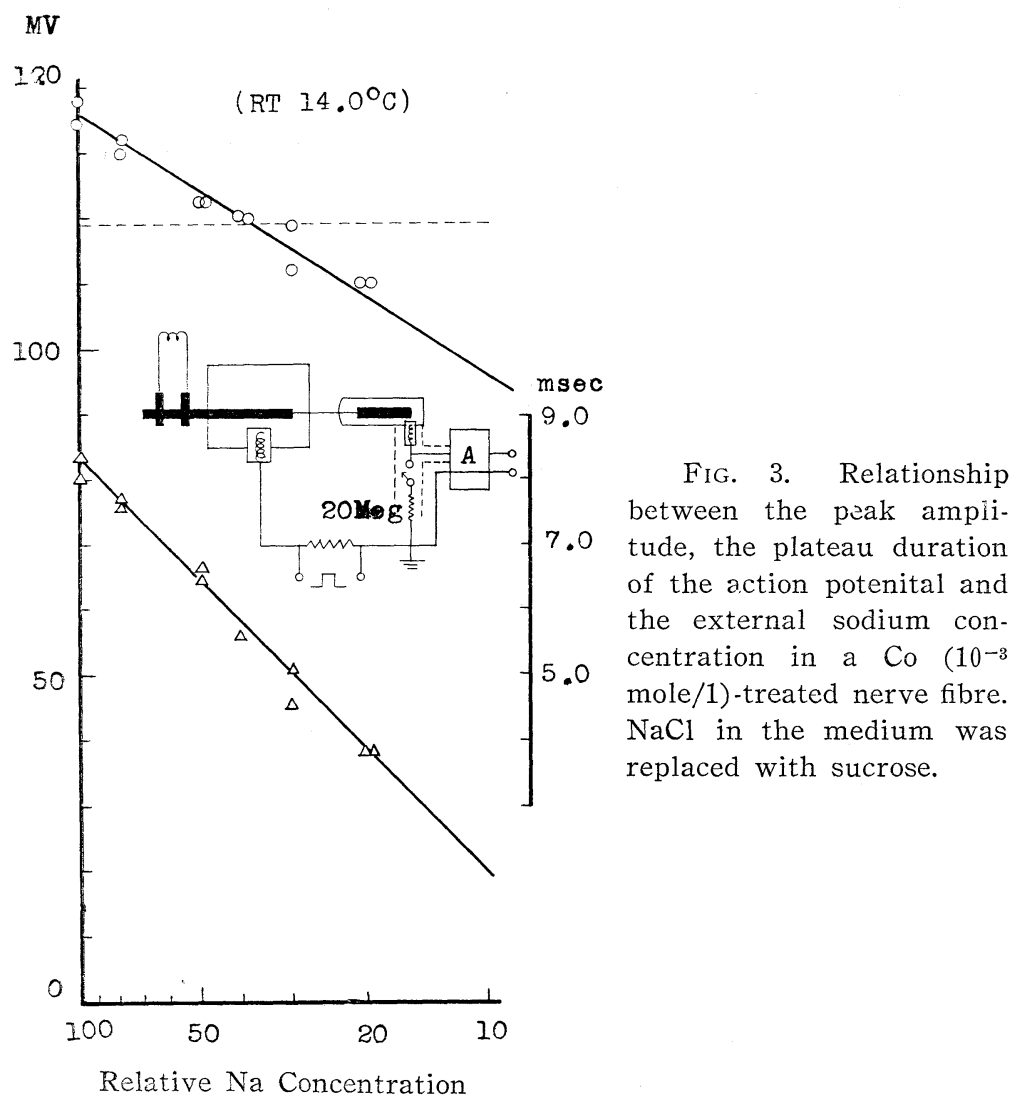

duration were changed linearly with proportion to the logarithm of sodium concentration. However, the duration of the plateau was more dependent upon the external sodium concentration than the peak voltage of the action potential. $K$ ion and plateau. There was some tendency in Co-treated fibres that changes in potassium concentration of the media caused not so large changes of the rheobase as observed in the normal fibres. Thus, higher concentrated potassium did not produce depolarization in some Co-treated fibres. In such preparations, high concentrated potassium prolonged the plateau duration. However, the prolongation in the plateau duration was likely to be induced by some critical potassium concentrations. It is seen in FIG. 4 that $13.5 \mathrm{mM}$ potassium chloride produced prolongation of the plateau-duration, while it did not so 


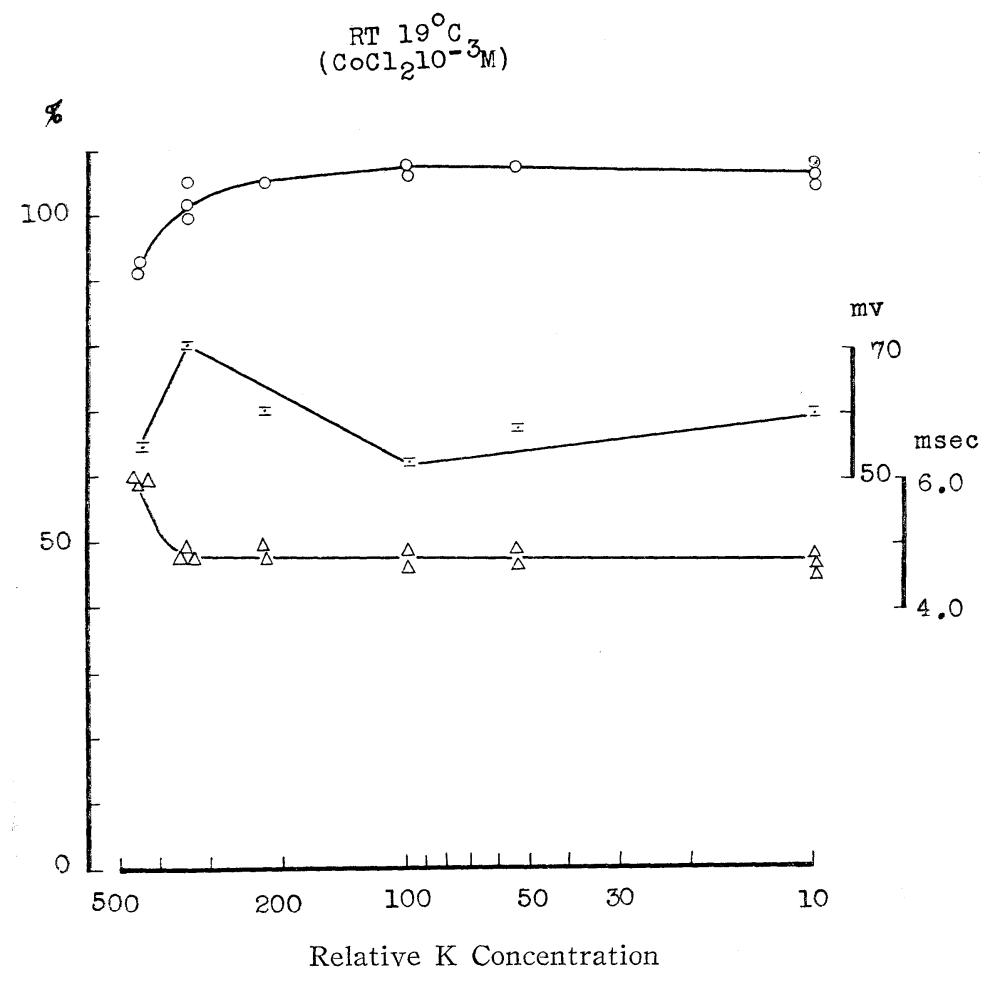

FIG. 4. Relationship between the rheobase, the spike height, the duration of the action current and the external potassium concentration (logarithmic scale) in a Co-treated nerve fibre.

large changes in the rheobase and the spike height. On the other hand, it was observed in another experiment that under the action of the same concentration of potassium chloride, the duration of the plateau was transiently prolonged at first and then curtailed after full development of depolarization. Calcium ion and plateau. The effects of calcium-deficiency on Co-treated nodes were (a) decrease in the rheobase, (b) reduction in the spike height and (c) further prolongation of the action current.

In FIG. 5 the effects of calcium-deficiency on a Co-treated node are illustrated. In this experiment, a single myelinated nerve fibre was previously exposed to the action of cobalt chloride. Then, the surrounding medium was replaced with $\mathrm{Ca}$-free Ringer and subsequently cobalt chloride was again added to the Ca-free medium. After washing out of cobalt chloride with Ca-free Ringer, the rheobase was decreased to a level which is usual in Ca-free media. However, the prolongation of action potential forming a plateau induced by Co ion did not disappear after washing. After addition of cobalt chloride to the Ca-free medium, the rheobase was increased to the normal level and the 
$\mathrm{CoCl}_{2}$ and $\mathrm{Ca}$ ion (R.T. $18^{\circ} \mathrm{C}$ )

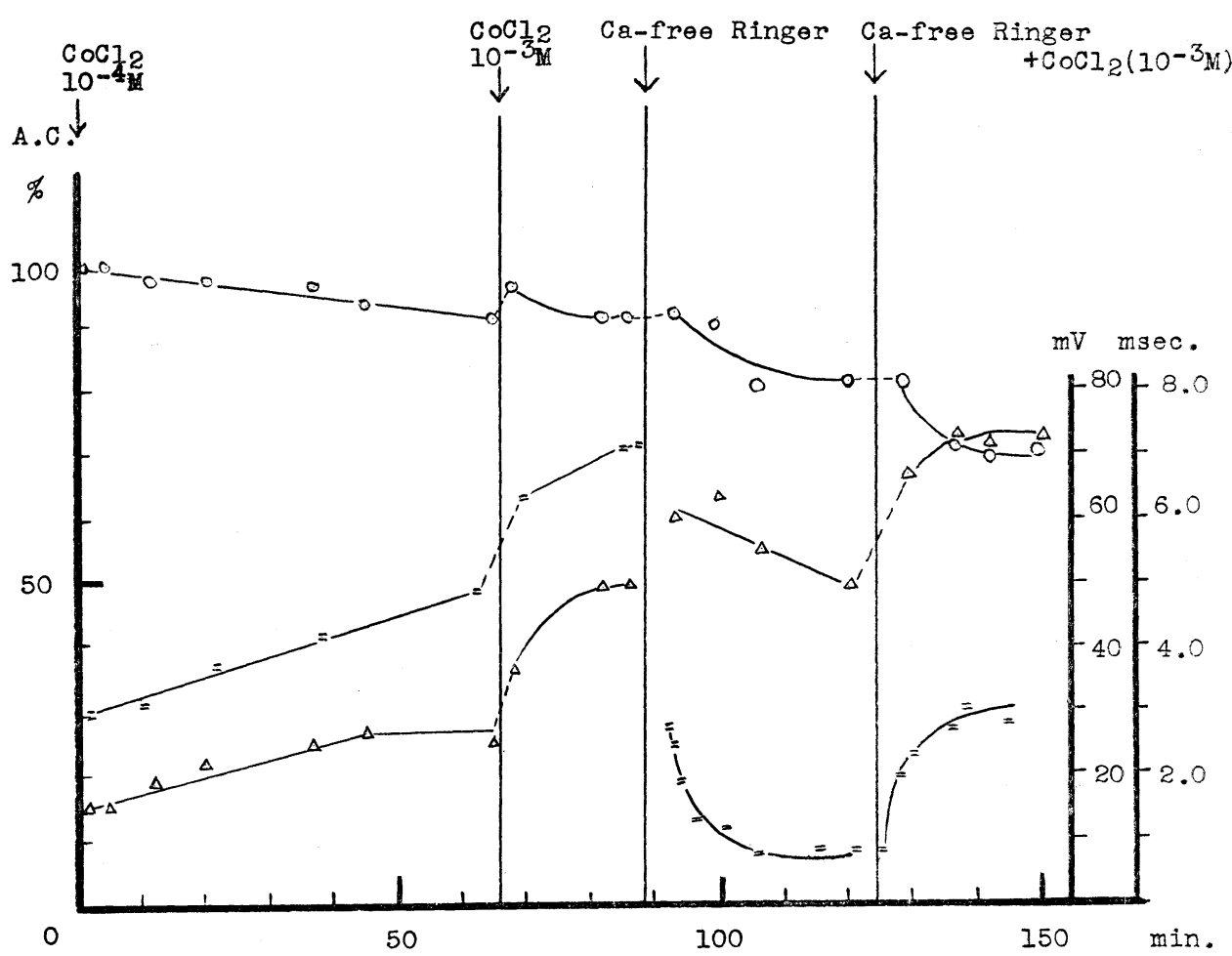

FIG. 5. Ca-deficiency and effects of cobalt ion.

duration of action current was further prolonged.

FIG. 6 illustrates a concentration-effect relationship of cobalt chloride in Ca-free media. In this experiment, the surrounding normal Ringer was replaced with Ca-free Ringer. Under the action of Ca-free Ringer, the rheobase was decreased profoundly and the spike height of the action current was reduced by about 30\%. Then, the medium was replaced with Ca-free Ringer containing various concentrations of cobalt chloride.

In Ca-free media, too, the rheobase was increased proportionally with the concentration of cobalt chloride. The duration of action current was prolonged under the action of Co ion, but the plateau-forming action of Co ion was extremely diminished in higher concentrations, as it was the case in normal Ringer. The curtailment of the plateau by higher concentrated Co ion was accompanied with increase in shoulder height, a level from which rapid repolarization begins. The reduction in spike height and the decrease in rheobase induced by $\mathrm{Ca}$-deficiency was corrected almost completely by equimolar Co ion. 


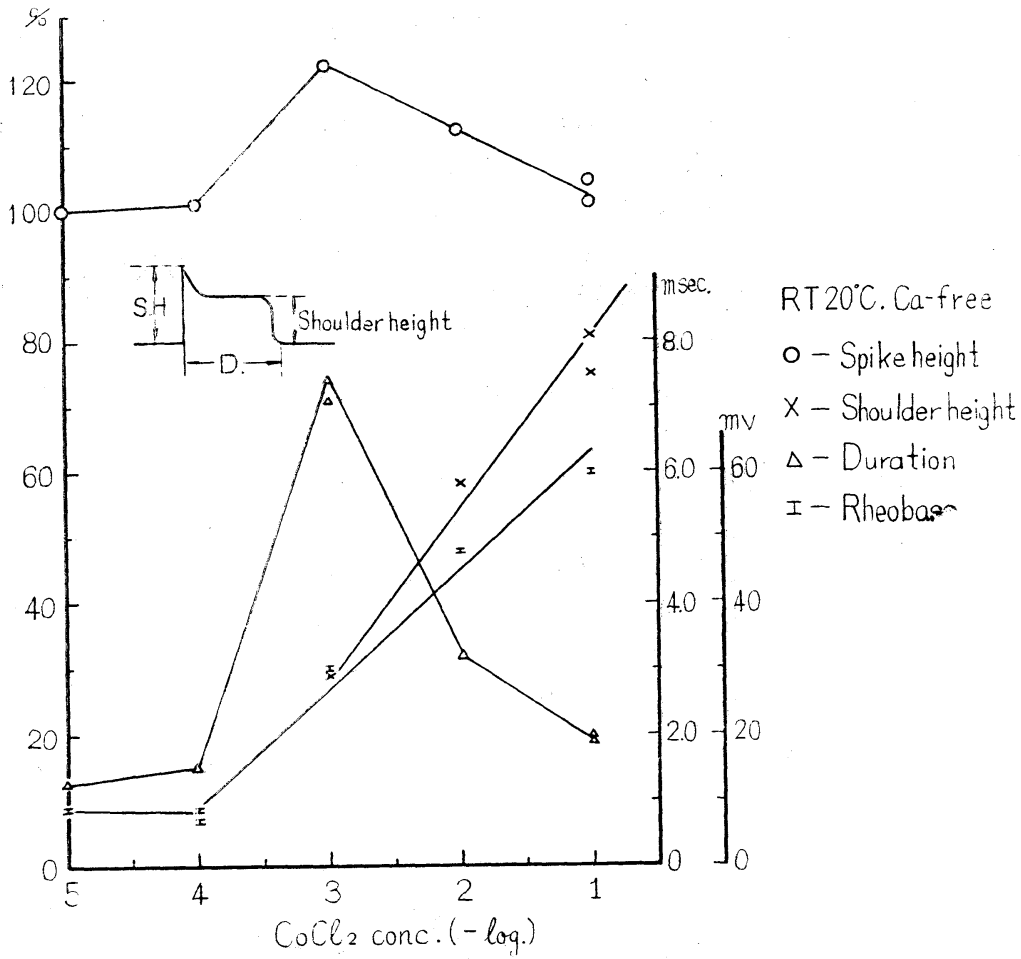

FIG. 6. Concentration-effect curve of cobalt chloride in the Ca-deficient medium

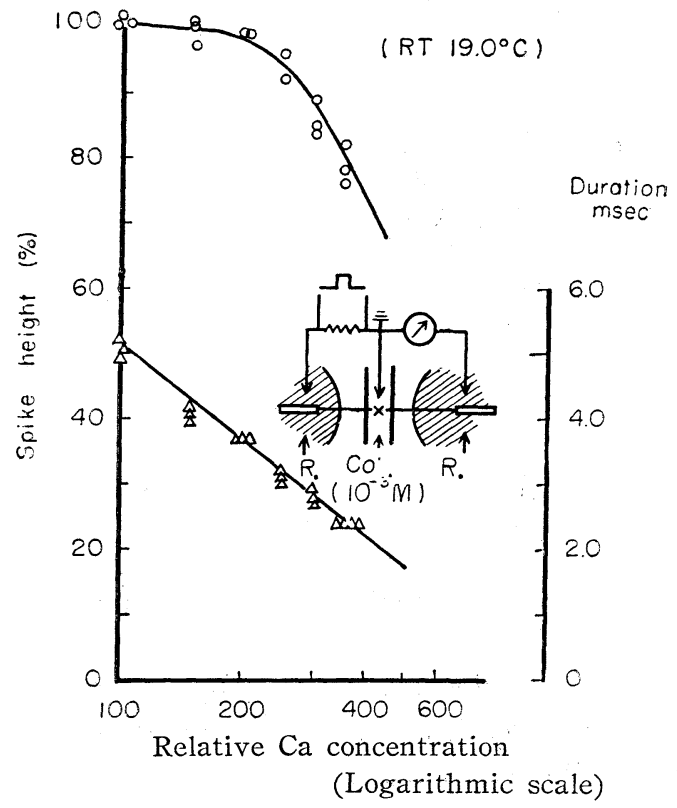

Fig. 7. Effects of higher concentrated calcium on the spike height and the duration of the action current of a Co $\left(10^{-3} \mathrm{~mole} /\right.$ 1)-treated nerve fibre. 
The effects of high concentrated calcium ion on Co-treated myelinated nerve fibres were (a) increase in the rheobase, (b) reduction in the spike height, (c) inhibition on the plateau of the action current and (d) increase in the shoulder height. An example of concentration-effect curves of calcium in Cotreated myelinated nerve fibres is furnished in FIG. 7.

In comparison with $\mathrm{Ca}$, the effects of $\mathrm{Mg}$ and $\mathrm{Ba}$ ion on the plateau formation in Co-treated fibres were preliminarily observed. $\mathrm{Mg}$ ion had a plateau-curtailing action similar to that of $\mathrm{Ca}$ ion, though its potency was much lower. On the other hand, $\mathrm{Ba}$ ion had no effect on the plateau of the action current induced by Co ion.

Narcotics and plateau. Narcotics, such as urethane or cocaine produced reduction in the spike height and increase in the rheobase of the normal fibre. They curtailed the duration of the action current only slightly. Co ion antagonized against the spike-height-reducing action of narcotics, though the

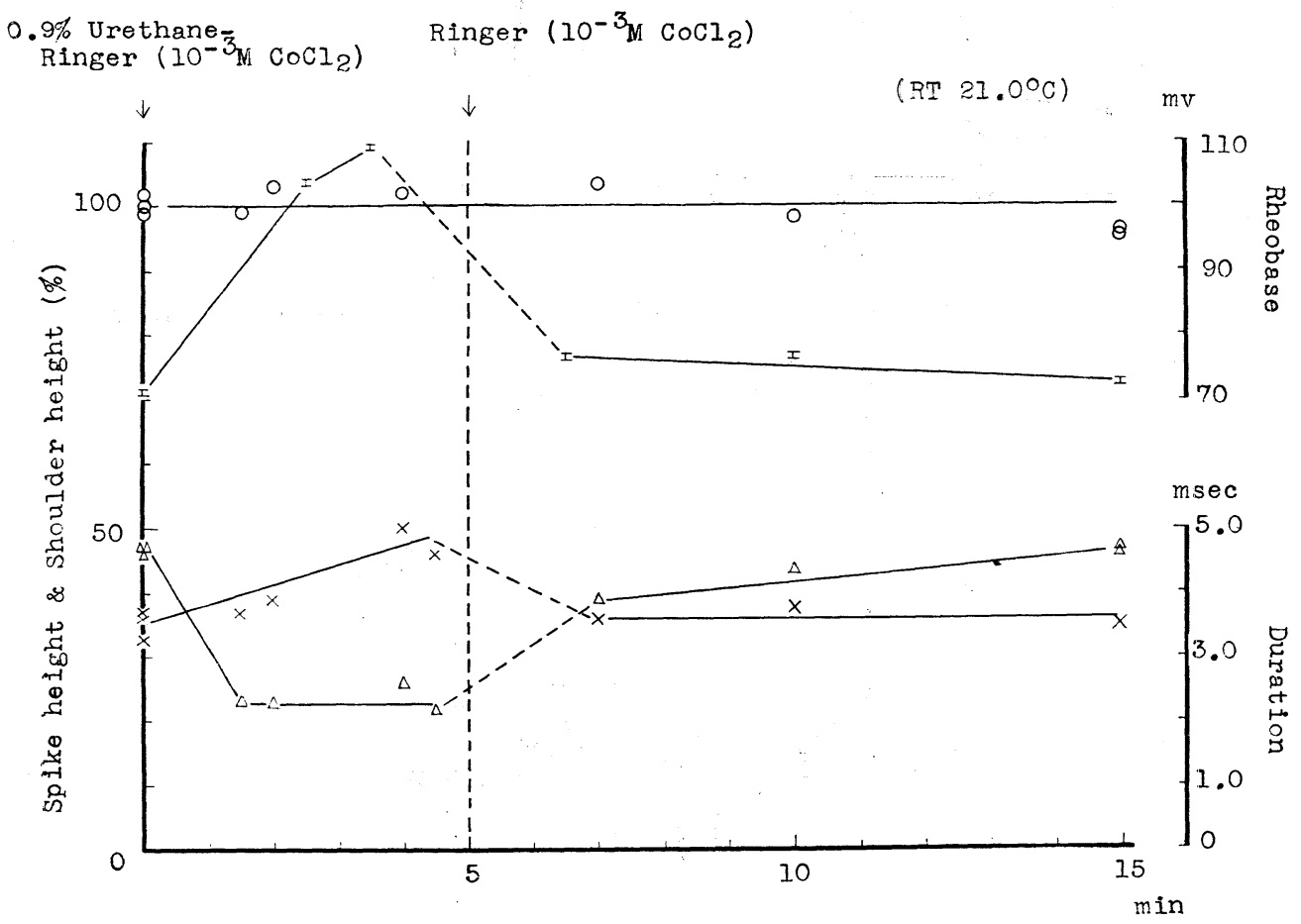

FIG. 8. Effects of urethane on a Co-treated nerve fibre.

antagonism between them varied considerably from preparation to preparation. In some Co-treated fibres, narcotics at suitable concentrations caused no change in the spike height, while they curtailed extremely the duration of the plateau (FIG. 8). 
However, narcotics produced usually curtailment of the plateau and reduction of the spike height at the same time, though the reduction of the spike height induced by narcotics was less in Co-treated fibres than in normal fibres. FIG. 9 illustrates the effect of cocaine on the action current of a single Co-treated nerve fibre.

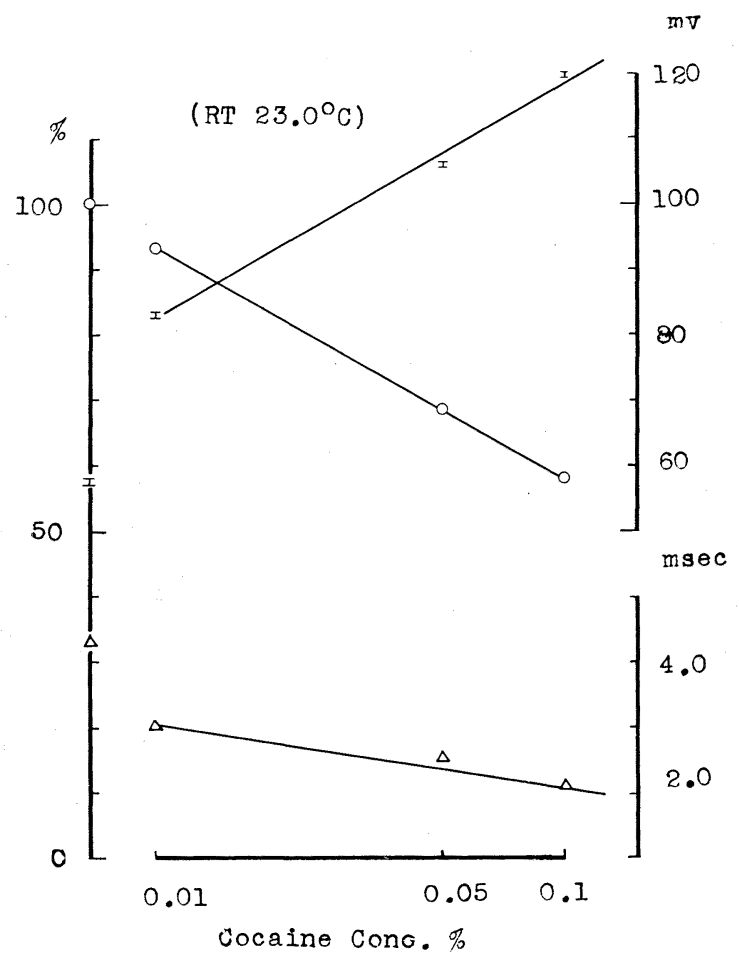

FIG. 9. Effects of cocaine on a Co-treated nerve fibre.

Electrotonus and plateau. Electrotonus produced some changes in the plateau of the action current of Co-treated nerve fibres. Catelectrotonus curtailed the duration of the plateau, increasing its slope. Under catelectrotonus, its shoulder became obscure. On the other hand, the duration of the plateau was not changed under anelectrotonus, although its slope became slower. Under anelectrotonus, the shoulder height was increased. It is seen in FIG. 10 that the plateau of the action current become clear under anelectrotonus both in Co-treated fibres and in normal fibres, although its duration was not so appreciably changed.

The spike height of the action current was reduced or increased progressively with increasing strength of cat- or anelectrotonus respectively in Co-treated fibres, as is the case with normal fibres (FIG. 11). The change in threshold or rheobase of Co-treated fibres under electrotonus was similar to 


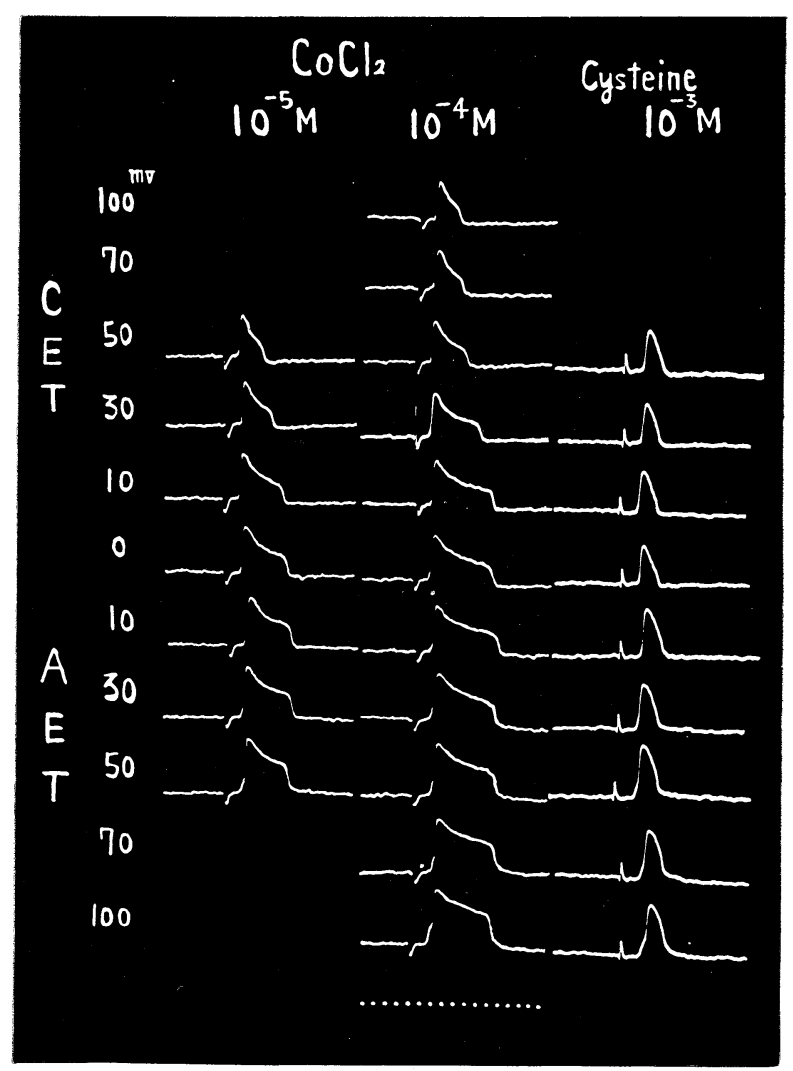

FIG. 10. Effects of electrotonus on the oscillogram of the action current in a Co-treated nerve fibre.

that of normal fibres.

Brief inward current pulse and plateau. When the pulse of anodal inward current was made short and applied during the plateau of the action current or potential, the portion of the action current or potential following the applied anodal pulse was abolished at a certain strength of the pulse in all-or-none manner.

The minimal intensity of the pulse needed for abolishing an action potential or current depended mainly upon the relative position of the pulse on the plateau. Much stronger pulses were needed in the early stage of the plateau than in its later stage.

On the other hand, the brief strong cathodal outward current applied through the active nodal membrane did not produce any change in the course of the activity.

These observations made about the Co- or Ni-treated nerve fibre accorded in general with the results of TASAKI and others ${ }^{5,6)}$ obtained in the normal 


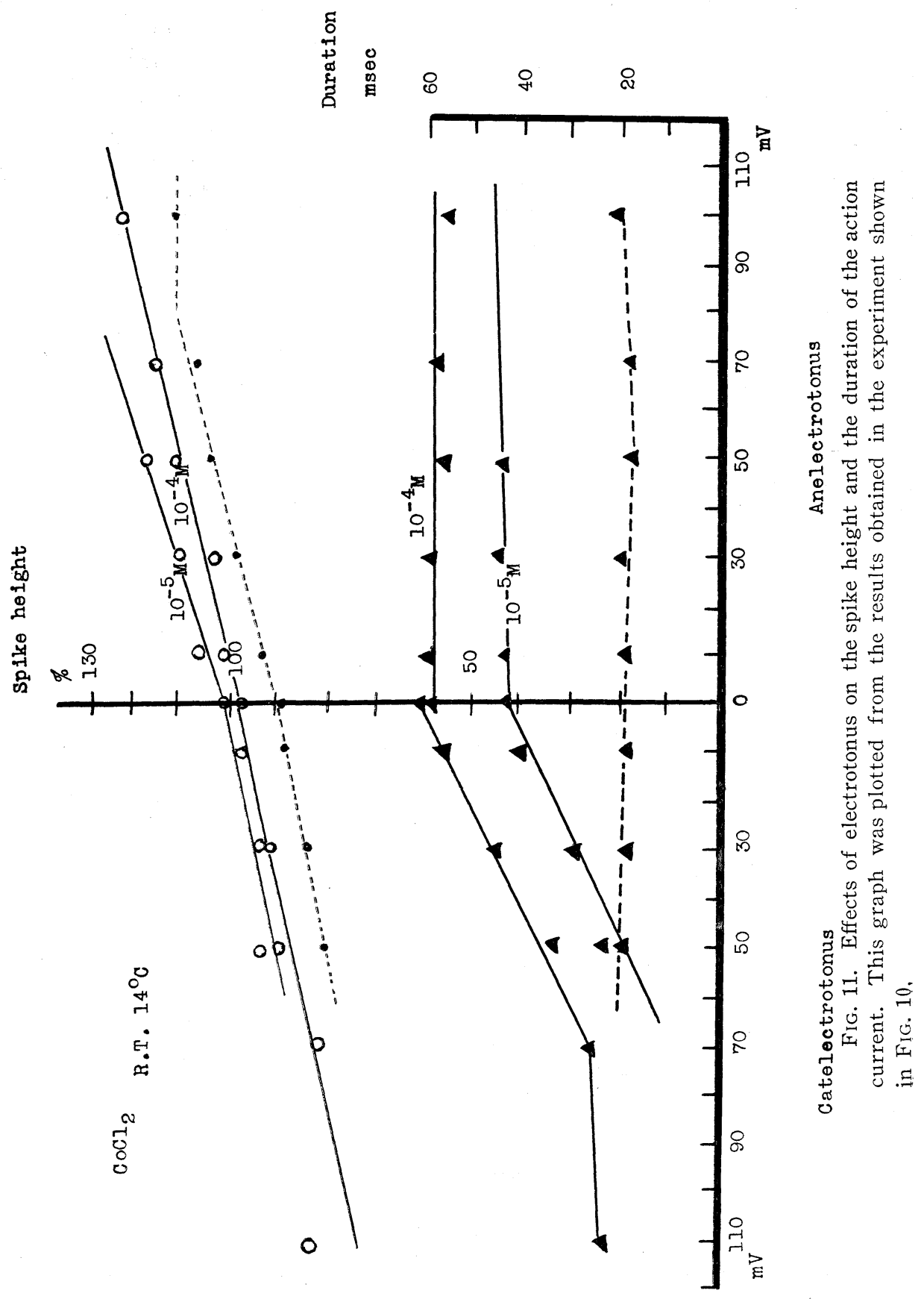


frog nerve fibre and in the tetraethylammonium-treated squid giant axon.

Plateau during the recovery phase. In Co-treated fibres, the recovery of the spike height and the duration of the action current was separable. The duration of the plateau was extremely curtailed during the relative refractory period and its recovery lagged remarkably behind that of the spike height. The recovery of threshold during the refractoriness was similar to that of the spike height. However, there appeared a marked supernormal phase following the refractory period. The excitability reached usually to $130-140 \%$ of the original value during this phase. During this supernormal phase, the duration of the plateau recovered gradually to the original value.

FIG. 12 illustrates the recovery phase of a single nerve fibre under the action of $10^{-4} \mathrm{~mole} / 1$ cobalt chloride. It is seen in this figure that the duration of the action current is about three fifths of the original value, while the spike height recovers completely. It takes about $50 \mathrm{msec}$. for the duration to recover completely, while the spike height recovers completely within 10 msec.

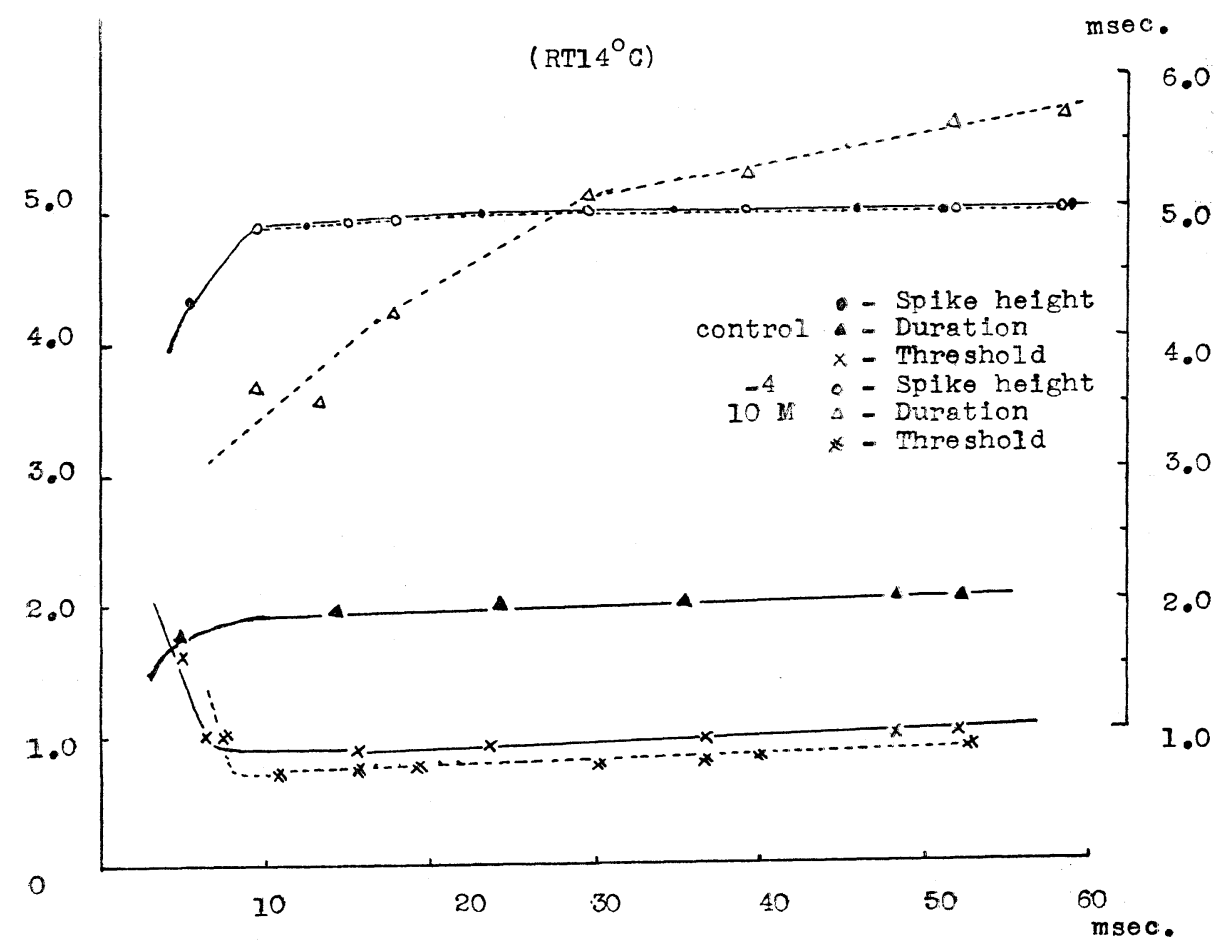

FIG. 12. Spike height, duration of the action current and threshold during the recovery phase in a Co-treated nerve fibre. Abscissa, time-interval from the start of the first stimulus to that of the second stimulus. As the safety factor in the transmission is in the normal fibre approximately 5 , the strength of action current from Co-treated resting node is taken to 5 times the threshold for the node. 
The time scales of these phenomena varied from preparation to preparation, for the actions of the agent varied considerably in degree. However, the general features of the recovery were the same in all the Co-treated fibres.

\section{DISCUSSION}

The excitation of the nodal membrane can be interpreted as some changes in the physico-chemical state of the membrane lipoprotein induced by a critical depolarization. TAKAHASHI et al. ${ }^{1,2}$ have found that these changes are profoundly affected by some modification of SH-groups of the nodal membrane induced with divalent transition metals. These findings suggest that the active state of the nodal membrane is associated with some spontaneously reversible perturbance of the membrane protein and these metals exaggerate this perturbance.

From the observation on the recovery phase of the spike height and the plateau duration, it seems reasonable to assume that the mechanism maintaining the active state of the nodal membrane is rather separable from that initiating it. The physico-chemical changes induced by divalent transition metals are rather concerned with the former mechanism. However, the action of these metals are not indifferent to the latter mechanism, since they counteract some actions of $\mathrm{Ca}$ ion or narcotics upon it.

On the other hand, the external sodium concentration controls both the spike height and the plateau duration. This phenomenon can be interpreted without contradiction as follows; the mechanism maintaining the active state is activated by sodium ions inflowing into the nodal membrane during its initial phase and there appears a parallelism between the amount of sodium ion inflow which is driven by the mechanism initiating the active state and the plateau duration.

In addition, $\mathrm{Co}$ or $\mathrm{Ni}$ ion counteracts the depolarizing action of potassium ion. Such effect of $\mathrm{Ni}$ ion was also observed by TASAKI ${ }^{i}$. However, we could not obtain so clear-cut evidence for any contribution of change in potassium conductance induced by these metals to the plateau formation.

Reducing the external calcium concentration $\left(\mathrm{Ca}_{0}\right)$, caused prolongation in the plateau duration, although it decreases the amplitude of the spike height. On the other hand, increasing $\mathrm{Ca}_{0}$, caused marked curtailment of the plateau duration, while it caused no change in the spike height within some range. Recently, Spyropoulos ${ }^{8)}$ observed the direct inhibiting action of $\mathrm{Ca}$ ion on the mechanism maintaining the active state. Therefore, its direct action seems to be more effective than the possible indirect action due to the changes in the activity of the sodium-carrier system ${ }^{9,10)}$.

The curtailing action of narcotics on the plateau seems to be due to their inhibiting action on the sodium-carrier system ${ }^{11}$. However, our findings suggest 
that they may act also directly on the mechanism maintaining the active state.

\section{SUMMARY}

In Co-treated or Ni-treated myelinated nerve fibres, the effect of various agents on the plateau of the action current was investigated.

1. Lithium could effect as a substitute for sodium in these fibres, too. Complete replacement of sodium with lithium produced reversibly an increase in the rheobase and only a slight curtailment of the plateau.

2. By replacement of $\mathrm{NaCl}$ with choline chloride, the plateau duration was curtailed. Spike height was reduced proportionally with the reduction in sodium concentration, while the curtailment of plateau duration was saturated under higher concentrated choline.

3. By replacement of $\mathrm{NaCl}$ with sucrose, both the spike height and the plateau duration were diminished linearly with proportion to the logarithm of sodium concentration. The plateau duration was more sensitive to changes in sodium concentration than the spike height.

4. Co or $\mathrm{Ni}$ ion counteracted the depolarizing action of potassium ion. High concentrated potassium prolonged the plateau duration without the depolarization. On the contrary, when it depolarized the nodal membrane, the plateau duration was curtailed. The reduction of potassium concentration did not cause any appreciable change in the plateau duration.

5. The deficiency of calcium ion in the surrounding media produced decrease in the rheobase, reduction in the spike height and prolongation of the plateau duration. Co or $\mathrm{Ni}$ ion augmented the spike height diminished by Ca-deficiency and increased the lowered rheobase. The increase in Ca concentration curtailed the plateau duration.

6. Narcotics, such as urethane or cocaine curtailed the plateau duration. As $\mathrm{Co}$ or $\mathrm{Ni}$ ion counteract the reducing effect of narcotics on the spike height, the curtailment sometimes occurred without appreciable change in the spike height under the action of their suitable concentrations.

7. Catelectrotonus curtailed the plateau duration, increasing its slope. On the other hand, under anelectrotonus its slope $b$ scame slower, but its duration was not changed.

8. Brief inward current applied during the plateau abolished the portion of the action potential following the application at a certain strength in all-ornone manner. The threshold intensity depended mainly upon the relative position of the pulse on the action potential. Much stronger pulses were needed in the early stage of the plateau than in its later stage.

9. During the relative refractory period, the spike height recovered as rapidly as in the normal fibre. However, the plateau duration did not completely recover until the end of the supernormal phase which became apparent after 
application of these metal ions.

10. Some characters of the excitation mechanism of the nodal membrane were discussed briefly.

This work was aided partly by grants from the Ministry of Education of Japan.

\section{REFERENCES}

1) Takahashi, H., Murai, T. and Sasaki, T. Plateau formation and sulphydryl groups in the plasma membrane. Nature, Lond. 182 : 1675-1677 (1958).

2) Takahashi, H., Murai, T. and Sasaki, T. Some chemical aspect of plateau formation in the action current of the myelinated nerve fibre. Jap. J. Physiol. 10: 280-291 (1960).

3) Brady, R., Spyropoulos, C. S. and TASAki, I. Intra-axonal injection of biologically active materials. Amer. J. Physiol. 194: 207-213 (1958).

4) TASAKI, I. and FRANK, K. Measurement of the action potential of myelinated nerve fiber. Amer. J. Physiol. 182: 572-578 (1955).

5) TASAKI, I. Initiation and abolition of the action potential of a single node of Ranvier. J. gen. Physiol. 39 : 377-395 (1956).

6) TASAKI, I. and Hagiwara, S. Demonstration of two stable potential states in the squid giant axon under tetraethylammonium chloride. J. gen. Physiol. 40: 859-885 (1957).

7) TASAKI, I. Demonstration of two stable states of the nerve membrane in potassium-rich media. J. Physiol. $148:$ 306-331 (1959).

8) Spyropoulos, C.S. Initiation and abolition of electric response of nerve fiber by thermal and chemical means. Amer. J. Physiol. 200 : 203-208 (1961).

9) Frankenhaeuser, B. and Hodgkin, A. L. The action of calcium on the electrical properties of squid axons.

10) Frankenhaeuser, B. The effect of calcium on the myelinated nerve fibre. $J$. Physiol. $137: 245-260$.

11) TAYLOR, R, E. Effect of procaine on electrical properties of squid axon membrane. Amer. J. Physiol. 196: 1071-1078 (1959). 